\title{
Method for the influence design assessment of a wheeled agricultural vehicle on the soil
}

\author{
Ekaterina Balakina*, Alexander Konshin, and Mikhail Kochetov \\ Volgograd State Technical University, Volgograd, Russian Federation
}

\begin{abstract}
The impact of a wheeled agricultural vehicle on the deformable support surface determines the vehicle's ability to move, as well as soil compaction, which is not desirable in agriculture. The agricultural machine must not cause more pressure on the ground than is permissible. Therefore, in the tasks of design numerical modelling of the agricultural vehicle movement or trailer, it is required to calculate the specified parameter. It is impossible to calculate without knowledge of the geometric characteristics of the contact spot associated with the normal deformation of the tire under normal load. To calculate these characteristics, it is necessary to have universal dependencies for determining the normal stiffness of the tire. These are available for tires of various purposes. The elastic properties of ultra-low pressure tires are insufficiently studied. Experimental studies of the elastic properties of these tires have been carried out with the authors participation. However, there are currently no dependencies to describe them. This does not provide the possibility of a correct design calculation of the influence of such tires on the soil. The purpose of the work: to develop a universal method for calculating the influence on the soil of agricultural vehicle. A universal method for calculating the impact of a wheeled agricultural machine on the ground has been developed. Universal design-experimental dependence for determining the normal stiffness of ultra-low pressure tires is obtained. It takes into account tire pressure, normal load under specific conditions and geometric characteristics.
\end{abstract}

\section{Introduction}

Tires influence on a deformable support surface determines the vehicle's ability to move, as well as soil compaction, which is not desirable in agriculture [1-4]. The agricultural vehicle must not cause more soil pressure. Therefore, in the tasks of design numerical modelling of the agricultural machine movement, it is required to calculate the specified parameter. It cannot be calculated without knowledge of the geometric characteristics of the contact patch [5-8, 9-12] associated with the normal tire deformation under normal load. To calculate these characteristics, it is necessary to have universal dependencies to determine the normal tire stiffness. These are available for tires of various purposes [13-15]. The elastic properties of ultra-low-pressure tires are not well understood. Experimental studies of the elastic properties of these tires have been carried out with the authors participation.

\footnotetext{
*Corresponding author: fahrgestel12011@yandex.ru
} 
However, there are currently no universal dependencies to describe them. This does not provide the possibility of a correct design calculation of the influence of such tires on the soil.

The aim of the research is the development a method for the influence design assessment of a wheeled agricultural vehicle on the soil.

\section{Research methods}

Has been investigated 7 ultra-low pressure tyres. In the course of the research, the load characteristics (the relationship between load and deformation) were measured at different tire pressures. They looked like pairs of experimental points $\left(P_{\mathrm{z}} ; Z\right)$. They were approximated and experimental dependences $P_{\mathrm{z}}=f(Z)$ were created. For example, the obtained dependencies are presented graphically in Figure 1.

Method is proposed for determination of coefficient of normal stiffness for ultra-low pressure tyre by initial experimental load characteristics $P_{\mathrm{z}}=f(Z)$ of different tyres. Its stages are shown below.

A. Approximation for each ultra-low-pressure tire of the experimental loading characteristics $P_{\mathrm{z}}=f(Z)$ by the same type functions for each pressure $p_{\mathrm{i}}$. As a result, we have for each tire a set of functions in the form $P_{\mathrm{z}}=a_{1} \cdot Z^{\text {bi }}$ for each pressure $p_{\mathrm{i}}$. Here $a_{\mathrm{i}}, b_{\mathrm{i}}-$ are constant coefficients.

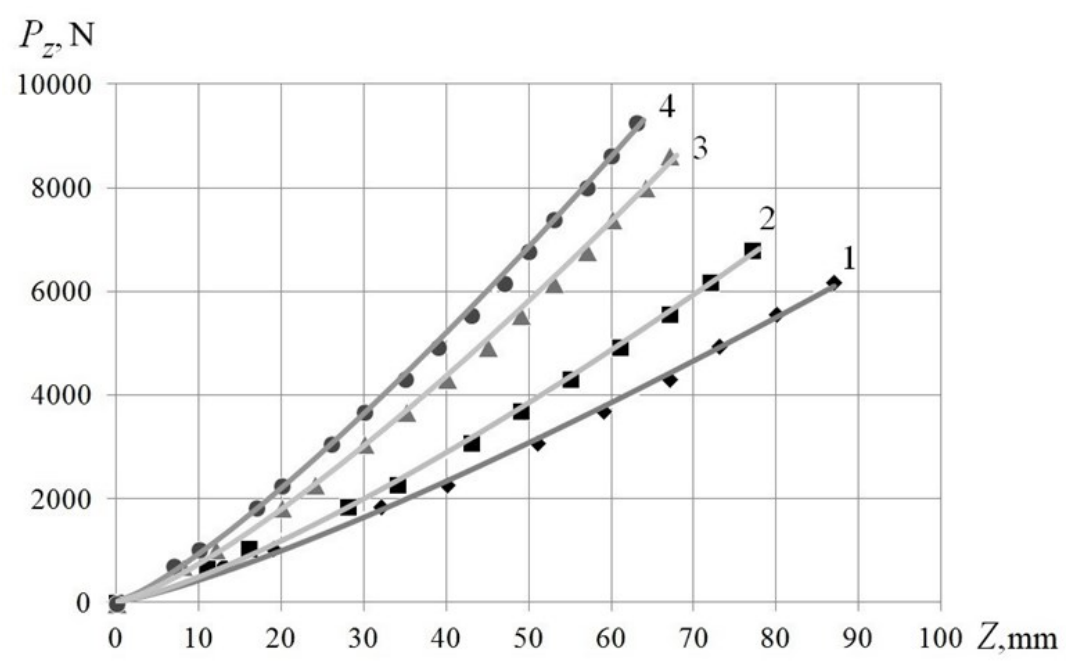

Fig. 1. Approximated experimental tire load characteristics 1300x600-533: $1-p=20 \mathrm{kPa}$; $2-p=30 \mathrm{kPa} ; 3-p=60 \mathrm{kPa} ; 4-p=70 \mathrm{kPa}$.

B. Approximation for each ultra-low pressure tire of the dependence for the obtained coefficients $a$ and $b$ on the pressure in the tire $p$. As a result, we get additional functions for each tire:

$$
a=f(p) \text { and } b=f(p)
$$

They have the form:

$$
a=k_{1} \cdot p ; b_{\approx} \text { const }
$$


As a result, for each ultra-low-pressure tire, the following expressions are obtained:

$$
P_{\mathrm{z}}=a \cdot Z^{\mathrm{b}} \text { или } P_{\mathrm{z}}=k_{1} \cdot p \cdot Z^{\mathrm{b}}
$$

C. Differentiating the normal load formula for normal deflection allows obtaining an expression for the normal stiffness of each ultra-low-pressure tire:

$$
C_{\mathrm{tz}}=\partial P_{\mathrm{z}} / \partial Z_{=} \partial_{(}\left(a \cdot Z^{\mathrm{b}}\right) / \partial Z=a \cdot b \cdot Z^{\mathrm{b}-1}=k_{1} \cdot p \cdot b \cdot Z^{\mathrm{b}-1}
$$

Introduction of symbols $k_{0}=k_{1} \cdot b ; d=b-1$ gives a new expression:

$$
C_{\mathrm{tz}}=k_{0} \cdot p \cdot Z^{\mathrm{d}}
$$

where $k_{0}=k_{1} \cdot b ; d=b-1$

D. Getting rid of the unknown value $Z$ on the right side of equation (5) for each ultralow-pressure tire:

$$
\begin{aligned}
& C_{\mathrm{tz}}=k_{0} \cdot p \cdot\left(P_{\mathrm{z}} / C_{\mathrm{tz})}^{\mathrm{d}}\right. \\
& C_{\mathrm{tz}}=k_{0}{ }^{1 /(1+\mathrm{d})} \cdot p^{1 /(1+\mathrm{d})} \cdot P_{\mathrm{z}}^{\mathrm{d} /(1+\mathrm{d})}
\end{aligned}
$$

Denote:

$$
\begin{aligned}
& k=k_{0}^{1 /(1+\mathrm{d})}=\left(k_{1} \cdot b\right)^{1 /[1+(\mathrm{b}-1)]}=\left(k_{1} \cdot b\right)^{1 / \mathrm{b}} \\
& n_{1}=1 /(1+d)=1 / b ; n_{2}=d /(1+d)=(b-1) / b
\end{aligned}
$$

As a result, expression (5) takes the general form:

$$
C_{\mathrm{tz}}=k \cdot p^{\mathrm{n} 1} \cdot P_{\mathrm{z}}^{\mathrm{n} 2}
$$

where $p$ - tire pressure; $P_{\mathrm{z}}-$ normal tire load; $k, n_{1}, n_{2}-$ constant coefficients characterizing the tire design.

Thus, it was possible to get rid of the unknown value of the normal deflection $Z$ in the formula (5) for calculating the coefficient of normal stiffness. Now this expression contains conditions: tire pressure $p$, normal tire load $P_{\mathrm{z}}$ and constant $k, n_{1}, n_{2}$, characterizing the tire design of this tire.

Table 1 shows the calculated constant coefficients for 7 models of ultra-low-pressure tires.

Table 1. Constant coefficients of expression (10)

\begin{tabular}{|c|c|c|c|c|c|c|c|}
\hline Tire number & $k_{1}$ & $b$ & $k_{0}$ & $d$ & $k$ & $n_{1}$ & $n_{2}$ \\
\hline 1 & 0.829 & 1.358 & 1.126 & 0.358 & 1.091 & 0.736 & 0.264 \\
\hline 2 & 0.738 & 1.263 & 0.934 & 0.263 & 0.947 & 0.792 & 0.208 \\
\hline 3 & 0.650 & 1.420 & 0.923 & 0.420 & 0.945 & 0.704 & 0.296 \\
\hline 4 & 0.675 & 1.380 & 0.931 & 0.380 & 0.949 & 0.725 & 0.275 \\
\hline 5 & 0.642 & 1.302 & 0.836 & 0.302 & 0.871 & 0.768 & 0.232 \\
\hline 6 & 0.695 & 1.336 & 0.929 & 0.336 & 0.946 & 0.749 & 0.251 \\
\hline 7 & 1.152 & 1.282 & 1.476 & 0.282 & 1.255 & 0.780 & 0.220 \\
\hline
\end{tabular}


E. Obtaining a universal dependence for determining the normal (radial) stiffness coefficient of an ultra-low-pressure tire.

Table 2 shows the geometric characteristics of the considered ultra-low-pressure tires and the obtained constant coefficients of the expression for calculating normal (radial) stiffness.

Table 2. Tire characteristics and obtained coefficients

\begin{tabular}{|c|c|c|c|c|c|c|c|c|}
\hline \multirow{2}{*}{$\begin{array}{c}\text { Tire } \\
\text { number }\end{array}$} & \multicolumn{4}{|c|}{ Tire characteristics } & \multicolumn{4}{c|}{ Obtained coefficients } \\
\cline { 2 - 9 } & {$\left[P_{\mathrm{z}}\right], \mathrm{N}$} & $D_{0}, \mathrm{~mm}$ & $H_{\mathrm{t}}, \mathrm{mm}$ & $B_{\mathrm{t}}, \mathrm{mm}$ & $H_{\mathrm{t}} / B_{\mathrm{t}}$ & $k$ & $n_{1}$ & $n_{2}$ \\
\hline 1 & 4000 & 1300 & 380 & 530 & 0.717 & 1.091 & 0.736 & 0.264 \\
\hline 2 & 6000 & 1300 & 380 & 600 & 0.633 & 0.947 & 0.792 & 0.208 \\
\hline 3 & 8000 & 1300 & 345 & 700 & 0.493 & 0.945 & 0.704 & 0.296 \\
\hline 4 & 8000 & 1300 & 345 & 700 & 0.493 & 0.949 & 0.725 & 0.275 \\
\hline 5 & 6000 & 1200 & 330 & 600 & 0.550 & 0.871 & 0.768 & 0.232 \\
\hline 6 & 8000 & 1300 & 345 & 700 & 0.493 & 0.946 & 0.749 & 0.251 \\
\hline 7 & 8000 & 1300 & 345 & 700 & 0.493 & 1.255 & 0.780 & 0.220 \\
\hline
\end{tabular}

Expressions are obtained

$$
\left\{\begin{array}{l}
k=6.1+17.4 \cdot H_{\mathrm{t}} / B_{\mathrm{t}}-14.6\left(H_{\mathrm{t}} / B_{\mathrm{t}}\right)^{2} \\
n_{1}=-0.67+4.82 \cdot H_{\mathrm{t}} / B_{\mathrm{t}}-4\left(H_{\mathrm{t}} / B_{\mathrm{t}}\right)^{2} \\
n_{2}=1.65+4.764 \cdot H_{\mathrm{t}} / B_{\mathrm{t}}-3.94\left(H_{\mathrm{t}} / B_{\mathrm{t}}\right)^{2}
\end{array}\right.
$$

where $H_{\mathrm{t}}$ - height of the tire profile in $\mathrm{mm} ; B_{\mathrm{t}}-$ width of the tire profile in $\mathrm{mm}$.

Figure 2 shows the results of calculating the coefficients $k, n_{1}, n_{2}$ by expressions (11) in graphical form. Points show the according coefficients values for different tires from Table 2.

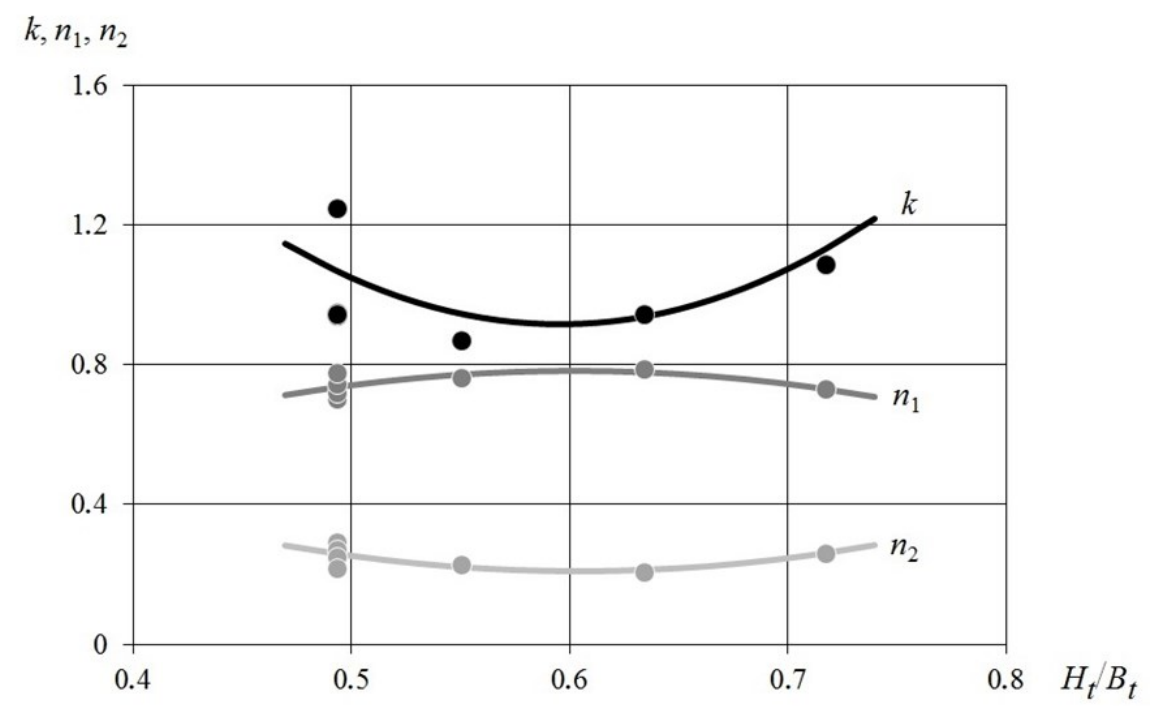

Fig. 2. Coefficients for calculating the radial stiffness of ultra-low-pressure tires: lines - calculation by approximated dependencies; points - experiment. 
After calculating the coefficients $k, n_{1}, n_{2}$ for a tire with a given ratio $H_{\mathrm{t}} / B_{\mathrm{t}}$, it becomes possible to calculate the radial stiffness of an ultra-low-pressure tire from the expression $C_{\mathrm{tz}}$ $=k \cdot p^{\mathrm{n} 1} \cdot P_{\mathrm{z}}^{\mathrm{n} 2}$.

\section{Results and discussion}

Thus universal calculation and experimental dependence (10) for ultra-low-pressure tires was obtained to determine the normal stiffness. It takes into account the tire pressure, the normal load under specific conditions and geometric characteristics. In the obtained dependence (10), the coefficients $k, n_{1}, n_{2}$ are functions of the section geometric characteristics of the profile $\left(H_{\mathrm{t}} / B_{\mathrm{t}}\right)$. The indicated coefficients can be calculated by expression (11).

Using the obtained dependencies, it is possible to determine the normal tire deformation and the geometric characteristics of the contact patch of the ultra-low-pressure tire with the soil, as well as the pressure on the soil.

Soil pressure

$$
p_{\mathrm{c}}=P_{\mathrm{z}} / F_{\mathrm{c}}
$$

where $F_{\mathrm{c}}-$ is the contour area of the contact spot.

According to the expressions obtained, it is possible to calculate the pressure on the soil - a particularly important parameter for these tires, because too much of its value may lead to obstruction of the agricultural vehicle movement or soil compaction, which is an undesirable factor in agriculture.

\section{Conclusion}

1. A universal method for calculating the influence on the soil of agricultural vehicle on ultra-low-pressure tires has been developed.

2. A universal calculated and experimental dependence is obtained to determine the normal stiffness of ultra-low-pressure tires. It takes into account the tire pressure, the normal load in the given conditions and the geometry. In the obtained dependence, three dimensionless coefficients are functions of the tire geometric characteristics: height and width.

\section{References}

1. V. I. Pryadkin, V. Y. Shapiro, Z. A. Godzhaev, Transport and technological machines on ultra-low-pressure tires (2019)

2. V. I. Kotlyarenko, S. V. Goncharenko, Z. A. Godzhaev, Tractors and agricultural machines, 2, 17-21 (2014)

3. Z. A. Godzhaev, S. V. Goncharenko, A. V. Artyomov, Tractors and agricultural machines, 3, 35-47 (2020)

4. Z. A. Godzhaev, A. Y. Izmailov, V. I. Pryadkin, Tractors and agricultural machines, 4, 14-17 (2014)

5. E. V. Balakina, V. N. Zadvornov, D. S. Sarbaev, I. V. Sergienko, Y. N. Kozlov, International Conference on Innovations in Automotive and Aerospace Engineering, IOP, 27 May - 1 June 2019, Irkutsk, Russia (2019) 
6. V. Y. Revenko, Z. A. Godzhaev, A. V. Rusanov, Tractors and agricultural machines, 5, 48-54 (2019)

7. Cristian MINCA, Review of the Air Force Academy, 1, 149-154 (2015)

8. S. Khaleghian, O. Ghasemalizadeh, S. Taheri, Tire Science and Technology, 44(4), 248-261 (2016)

9. F. Leiva-Villacorta, A. Vargas-Nordcbeck, J. P. Aguiar-Moya, LanammeUCR (2016)

10. P. Tomaraee, Spanish Journal of Agricultural Research, 13(2) (2015)

11. V. Ivanov, Advances in Tribology (2010)

12. D. Woodward, 4th International Safer Roads Conference (2014)

13. E. V. Balakina, V. N. Zadvornov, M. S. Berezovsky, Automotive industry, 8, 7-10 (2020)

14. V. I. Salnikov, A. A. Barashkov, V. N. Zadvornov, Automotive industry, 7, 13-14 (2014)

15. V. N. Zadvornov, E. V. Balakina, N. A. Mishenkov, Journal of Friction and Wear, 41(4), 354-358 (2020) 